\title{
Multiple Immunoreactive Forms of Osteocalcin in Uremic Serum
}

Caren M. Gundberg and Robert S. Weinstein

Laboratory of Human Biochemistry, Children's Hospital and Harvard School of Dental Medicine, Boston, Massachusetts 02115; Metabolic Bone Disease Laboratory, Department of Medicine, Medical College of Georgia, Augusta, Georgia 30912

\begin{abstract}
Circulating osteocalcin, which normally reflects the rate of bone formation, is elevated in uremia. In 18 patients receiving maintenance hemodialysis, serum osteocalcin levels were directly related to the bone formation rate $(r=0.88, P<0.001)$, osteoblastic osteoid surface density $(r=0.65, P<0.01)$, and osteoclastic resorptive surface density $(r=0.75, P<0.001)$. Multiple regression analysis showed that osteocalcin levels remained positively correlated with osteoclastic resorption when the bone formation rate was held constant $(P<0.01)$. The intimation that the coupling of bone formation and resorption could not explain the relationship between osteocalcin and resorption led us to determine whether fragments of this abundant matrix protein are released by bone resorption and retained in uremia. Sera from dialysis patients with renal osteodystrophy were fractionated by sequential gel filtration and HPLC, and assayed for immunoreactive osteocalcin. When normal serum was analyzed, a single sharp peak was found. In pooled sera from patients with high osteoclastic resorptive surfaces identified by histomorphometry, we found five additional immunoreactive peaks, while three additional peaks were detected in sera from patients with lower osteoclastic surfaces. Bio-Gel P-10 chromatography showed that these multiple peaks were of lower molecular weight than intact osteocalcin. We suggest that the liberation of bone matrix by osteoclasts contributes to the circulating osteocalcin immunoreactivity in uremia.
\end{abstract}

\section{Introduction}

In recent years, several noncollagenous proteins have been implicated in the regulation of skeletal metabolism (1). One of these proteins, osteocalcin, is synthesized by osteoblasts (2), comprises $20 \%$ of the noncollagenous protein in bone (3), and contains two to three residues of the calcium-binding amino acid, gamma carboxyglutamic acid $(\mathrm{Gla})^{1}$ (4). Although Gla residues cause osteocalcin to bind tightly to hydroxyapatite (5), nanomolar concentrations can be detected in serum by ra-

A preliminary report of this work was presented at the Southern Section Meeting of the American Federation for Clinical Research, New Orleans, LA, January 1985, and at the Annual Meeting of the American Society for Bone and Mineral Research, June 1985.

Address reprint requests to Dr. Gundberg, Children's Hospital, 320 Longwood Avenue, G1224, Boston, MA 02115. 1986.

Received for publication $19 \mathrm{July} 1985$ and in revised form 10 January

1. Abbreviations used in this paper: Gla, gamma carboxyglutamic acid; HPLC, high performance liquid chromatography.

J. Clin. Invest.

(c) The American Society for Clinical Investigation, Inc.

0021-9738/86/06/1762/06 \$1.00

Volume 77, June 1986, 1762-1767 dioimmunoassay (6). Increased osteocalcin levels are found in the circulation of patients with disorders characterized by increased bone turnover (7-9) and are normal in nonazotemic patients with nonosseous diseases (10). Osteocalcin has, therefore, become regarded as specific marker for bone formation (11).

Osteocalcin in serum has been thought to be derived exclusively from newly synthesized protein rather than from bone resorption, because within $3 \mathrm{~h}$ of exposure to vitamin $\mathrm{K}$-antagonism by warfarin, serum osteocalcin is devoid of Gla, while bone osteocalcin is fully gamma carboxylated (12). During bone resorption, the protein may be degraded by osteoclasts to nonor weakly immunoreactive fragments. Osteocalcin is cleared rapidly by glomerular filtration (12) and serum levels of immunoreactive osteocalcin are elevated in patients with renal insufficiency (13). In patients with renal osteodystrophy, the increased osteocalcin levels have been shown to reflect decreased renal clearance and increased bone formation $(13,14)$. If, however, fragments of osteocalcin with the appropriate antibody recognition site were released from bone during resorption, these fragments could accumulate and be detected in patients with renal failure. Bone resorption would thus also contribute to the increased serum osteocalcin concentrations found in uremia.

The present study shows that serum osteocalcin levels in dialysis patients are directly related to both bone formation rates and osteoclastic resorptive surfaces and demonstrates the existence of immunoreactive fragments of osteocalcin in uremic sera.

\section{Methods}

Subjects. The study group consisted of 18 patients on maintenance dialysis for $4.6 \pm 0.8$ (SE) years (range, 1 to $13 \mathrm{yr}$ ). The mean age was $43.1 \mathrm{yr}$ (range, 23 to 70 years). There were 8 women and 10 men. Renal failure was from hypertension in 11 patients, chronic glomerulonephritis in 5 , and interstitial nephritis in 2 . Subjects with bone pain, fractures with trivial trauma, radiographic progression of renal osteodystrophy, or hypercalcemia were selected for study from a group of 50 dialysis patients. Patients were excluded from the study if they had received supplemental calcium, pharmacological doses of vitamin D (>10 $\mu \mathrm{g} / \mathrm{d})$, calcitonin, fluoride, estrogen, glucocorticoid, diphosphonate, coumarin or anticonvulsant drug therapy, or had parathyroid surgery, diabetes mellitus, hepatic cirrhosis, or steatorrhea. 19 subjects who were eligible agreed to bone biopsy. For technical reasons, one biopsy could not be quantitated and this patient's data was deleted from the study. Daily calcium intake in the study group averaged $400 \mathrm{mg} / \mathrm{d}$ and $10 \mu \mathrm{g}$ of vitamin $D_{2}$ was given in a daily multivitamin capsule. Phosphate-binding gels were prescribed, but compliance was poor. The patients were dialyzed in the hospital for 3-5 $\mathrm{h}$, three times a week, with a deionized dialysate containing $3.25 \mathrm{mEq} /$ liter calcium, $1.0 \mathrm{mEq} /$ liter magnesium, and $<15 \mu \mathrm{g} /$ liter aluminum. Informed consent was obtained in accordance with the guidelines of the Human Assurance Committee of the Medical College of Georgia.

Bone biopsy. The subjects received $750 \mathrm{mg} / \mathrm{d}$ of oral tetracycline $\mathrm{HCl}$ $23,22,21,6,5$, and $4 \mathrm{~d}$ before biopsy in the outpatient clinic. Transileal bone biopsies with a cortex at each end were obtained with a trephine of 5 or $7 \mathrm{~mm}$ i.d. Specimens were fixed at $10^{\circ} \mathrm{C}$ for $20 \mathrm{~h}$ in phosphate- 
buffered $10 \%$ formaldehyde containing $0.5 \%$ sucrose, dehydrated in graded acetone solutions, and embedded in methyl methacrylate without prior decalcification.

Histomorphometry. Longitudinal sections taken from one-third and one-half the thickness of each specimen were cut with a Jung model $\mathrm{K}$ heavy-duty microtome (15). Undecalcified $5-\mu \mathrm{m}$ thick sections were stained by a modification of the Masson technique and evaluated histometrically with a Merz-Schenk graticule (Klarmann Rulings, Inc., Manchester, $\mathrm{NH}$ ) at $\times 250$ magnification (numerical aperture, 0.60 ). A mean area of $21 \mathrm{~mm}^{2}$ (117 fields) was examined. Several sections were decalcified for examination with polarized light. The tetracycline labels were measured on unstained $20-\mu \mathrm{m}$ thick sections with a linear graticule (Wild-Heerbrugg Instruments, Inc., Farmingdale, NY) viewed by epifluorescence (16). The following histological features were quantitated: trabecular bone volume (TBV) expressed as the percentage of whole tissue including marrow and bone, relative osteoid volume (ROV) expressed as the percentage of trabecular bone, osteoid surface (OS) expressed as the percentage of trabecular surface, osteoblastic osteoid surface (OB) expressed as the percentage of trabecular surface, osteoblastic osteoid surface density (SVob) expressed as the decimal fraction of osteoblastic osteoid surface multiplied by both the surface density (SV) in square millimeters per cubic centimeter of tissue and the scaling factor of $4 / \pi$ $(17,18)$, mean osteoid seam width (Seam) obtained by dividing the total osteoid volume by the osteoid surface density (18), osteoclastic resorptive surface (OCL) expressed as the percentage of trabecular surface, osteoclastic resorptive surface density (SVocl) expressed as the decimal fraction of osteoclastic resorptive surface $\times \mathrm{SV}$, peritrabecular marrow fibrosis (f) expressed as the percentage of trabecular surface covered by fibrosis tissue, and aluminum surface (Al) expressed as the percentage of total surface that forms a bright red band with the ammonium salt of aurine tricarboxylic acid (19).

Mineral appositional rate (MAR, micrometers per day) was calculated as the mean distance between the midpoints of the double tetracycline labels divided by the interdose duration. All the double-labeled and half the single-labeled trabecular surface were used to determine the fractional extent of active mineralization (LAB). The corrected mineral appositional rate (CMAR, micrometers per day) was calculated as MAR $\times$ LAB divided by the osteoid surface $\times 100$. Mean osteoid seam width divided by CMAR gave the corrected mineralization lag time (CMLT, days). Bone formation rate per unit tissue per year (BFR-T, percent per year) was calculated as MAR $\times \mathrm{LAB} \times 365 \times \mathrm{SV} \times \mathrm{TBV} \times 10^{-4}(20,21)$. All measurements excluded the endosteal borders of the cortices by a distance of two graticule spaces. The slides were examined without knowledge of the serum osteocalcin concentrations.

Reference values were calculated from undecalcified specimens of iliac crest obtained from 33 individuals at autopsy after sudden death (mean age $49.5 \mathrm{yr}$; range, 20 to $80 \mathrm{yr}$ ). There were 15 women and 18 men. The embedded blocks were kindly supplied by Dr. B. L. Riggs. The normal tetracycline data are from four healthy volunteers.

Chromatography. Fasting serum was obtained on the morning of bone biopsy. Osteocalcin was determined by radioimmunoassay as previously described (22). Serum samples from normal adults or from the dialysis patients were fractionated by gel exclusion chromatography followed by high performance liquid chromatography (HPLC). 1-2 ml of serum from each of five patients with high osteoclastic resorptive surfaces (patients 1, 3, 7, 8, and 11; Table I) was pooled for one fractionation. 1-2 $\mathrm{ml}$ of serum from each of five patients with low osteoclastic resorptive surfaces (patients 13,14,16,17, and 18) was pooled for a separate analysis. Serum from three normal adults was analyzed separately.

5-10 ml of serum were placed on a Sephadex G-100 column (15 $\times 100 \mathrm{~cm}$ ) (Pharmacia Fine Chemicals, Uppsala, Sweden), eluted with 3 liters of $50 \mathrm{mM} \mathrm{NH} \mathrm{CHO}_{3}$, and 5-ml fractions were collected. The fractions containing immunoreactive osteocalcin were pooled, lyophilized, and reconstituted in $1 \mathrm{ml}$ of $50 \mathrm{mM} \mathrm{NH}_{2} \mathrm{HCO}_{3}$. The reconstituted fractions were then applied to a Bio-Gel P-10 column $(1.5 \times 110 \mathrm{~cm})$ (Bio-Rad Laboratories, Richmond, CA) and eluted with the same buffer. The Sephadex fractions with immunoreactive osteocalcin were also characterized by HPLC using a Waters $\mathrm{C}_{18} \mu$ Bondapak reverse-phase column (Millipore Corp., Milford, MA). The samples were eluted with a 2-ml/min linear $30-70 \%$ solvent B in solvent A gradient [solvent A $=0.1 \%$ (vol/vol) tetrafluoroacetic acid (TFA) (Pierce Chemical Co., Rockford, IL); solvent B $=0.1 \%$ TFA in $70 \%$ acetonitrile (Burdick and Jackson Laboratories Inc., Muskegon, MI)]. Fractions ( $0.1 \mathrm{ml})$ were collected and assayed for osteocalcin. Synthetic or enzymatically produced fragments $(100-200 \mu \mathrm{g})$ were analyzed individually by HPLC. The elution of each fragment was monitored by absorbance at 210 or $276 \mathrm{~nm}$.

Preparation of fragments. Fragments corresponding to residues $41-$ $49,39-49$, and 35-49 of bovine osteocalcin were synthesized by the Merrifield solid phase technique (23) with a Beckman automatic peptide synthesizer (Beckman Instruments, Inc., Fullerton, CA). Each fragment was purified by gel filtration with a Bio-Gel P-2 column. Purity was confirmed by HPLC and amino acid analysis.

Fragments 1-19, 1-40, 20-43, and 45-49 from bovine osteocalcin were prepared by trypsin or carboxypeptidase $Y$ digestion as previously described (24). Individual peptides from the enzymatic digests were separated by HPLC and identified by amino acid analysis.

The immunoreactivity of each fragment in the standard radioimmunoassay was determined by assaying $0.3 \mathrm{ng} / \mathrm{ml}$ to $3.0 \mu \mathrm{g} / \mathrm{ml}$ of peptide serially diluted in $10 \mu \mathrm{l}$ of charcoal-absorbed human serum.

Statistical analysis. Values are expressed as mean \pm standard error. The $\mathbf{5 - 9 5 \%}$ confidence limits are displayed where appropriate. Relationships between different variables were calculated by linear regression analysis. Multivariate relationships were analyzed by stepwise multiple regression analysis (25).

\section{Results}

Histomorphometry. The osteocalcin levels and histomorphometric data are shown in Table I. There was the expected wide spectrum of bone turnover typical of renal osteodystrophy (26). Patients 1-5 showed accelerated bone formation rates when compared with normal subjects $(>6.60 \% / y r)$, while patients $13-$ 18 had subnormal rates $(<0.70 \% / y r)$. Accelerated bone turnover was characterized by an augmented trabecular bone volume with an abundance of woven bone. Relative osteoid volume and osteoid surface, osteoblastic osteoid, osteoclastic resorptive, and fibrosis-lined surfaces were prominent. Aluminum-stained trabecular surface and aluminum-stained cement lines were found in some of the patients with accelerated bone formation. In these patients, double tetracycline labels were sometimes noted at sites that stained positively for aluminum (19). Decreased bone turnover was characterized by extraordinarily increased osteoid volume, surface, and seam thickness. Lamellar bone was prevalent. When compared with the biopsies from patients with accelerated bone turnover, there was a relative paucity of osteoblastic osteoid and osteoclastic resorptive surfaces, although osteoblastic and osteoclastic surfaces were often greater than normal. Aluminum stained trabecular surface was detected in all the patients with reduced bone turnover.

Serum osteocalcin. The osteocalcin levels ranged from 12 to $317 \mathrm{ng} / \mathrm{ml}$, values 1.7 to 45 times the normal mean $(7.1 \pm 2.5$ SD). Serum osteocalcin levels were directly related to the bone formation rate per unit volume of tissue per year $(r=0.88, P$ $<0.001$ ) (Fig. 1). The relationship between osteocalcin levels and the bone formation rate was still significant when patients with bone formation rate values of zero were deleted $(r=0.86$, $P<0.001)$. The serum osteocalcin levels were also directly related to the corrected mineral appositional rate $(r=0.58, P<0.01)$, to the osteoblastic osteoid surface density $(r=0.65, P<0.01)$, and to the osteoclastic resorptive surface density $(r=0.75, P$ $<0.001)$. Osteoblastic osteoid and osteoclastic resorptive surface were positively correlated $(r=0.49, P<0.05)$ and the osteoblastic 


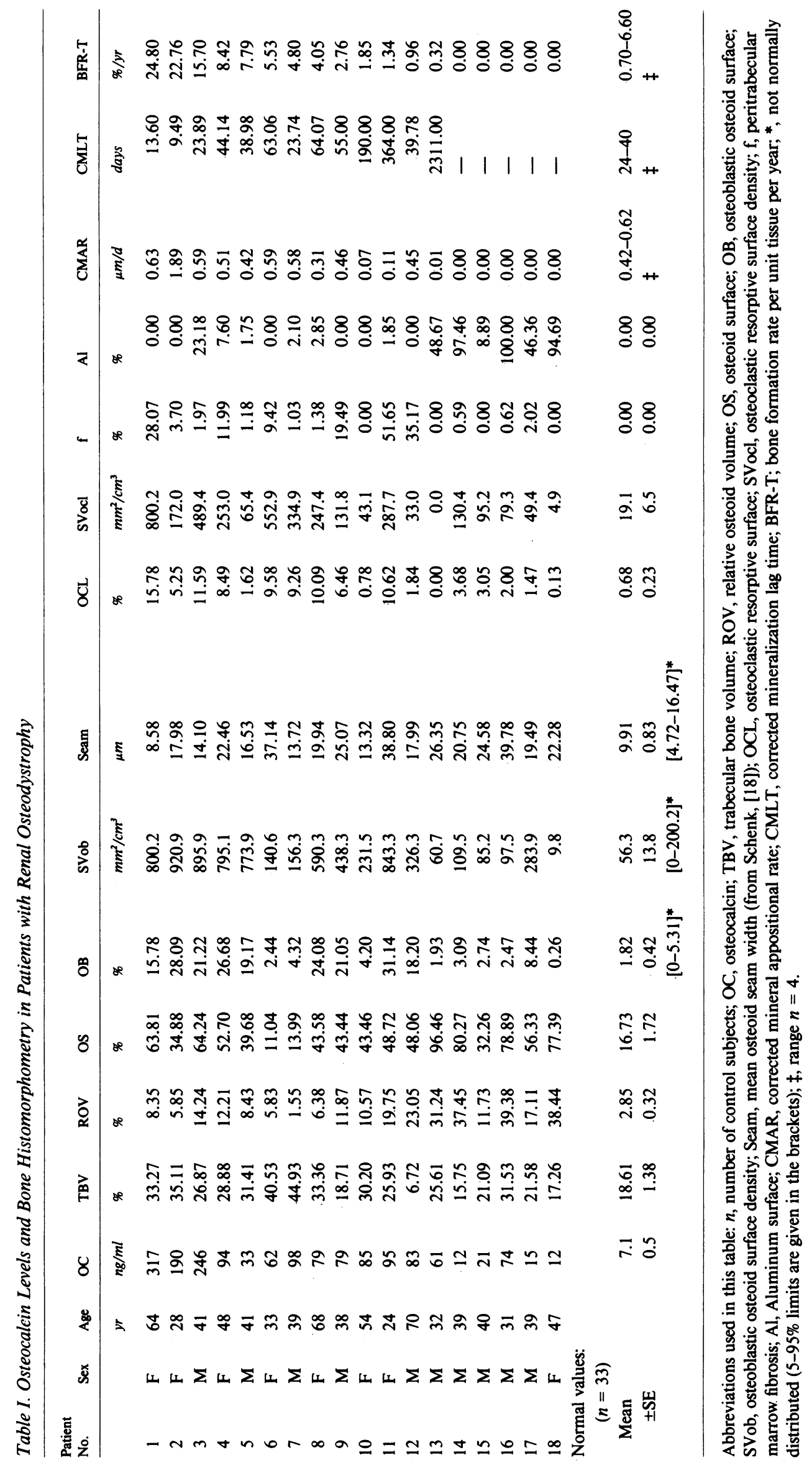




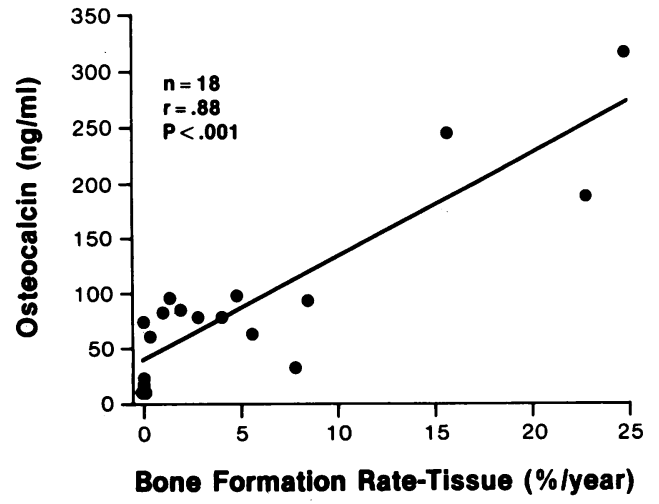

Figure 1. Regression analysis of serum osteocalcin levels and bone formation rate per unit tissue in 18 dialysis patients with renal osteodystrophy.

osteoid and osteoclastic resorptive surface density measurements directly related to the bone formation rate $(r=0.73, P<0.001$ and $r=0.68, P<0.01$, respectively).

Because bone formation and resorption are coupled in renal osteodystrophy $(14,26)$, the positive correlation between serum osteocalcin and osteoclastic resorptive surface was expected. We used stepwise multiple regression analysis to determine if there was a portion of the relationship between osteocalcin levels and bone resorption that was independent of coupling. When bone formation rates were held constant, the positive relationship between osteocalcin levels and osteoclastic resorption persisted $(P$ $<0.01)$. A majority of the variation in osteocalcin concentrations (78\%) was accounted for by changes in the bone formation rate with an additional $4.0 \%$ due to changes in osteoclastic resorptive surface density.

Antibody specificity. Fragments 1-19, 20-43, and 45-49 derived from tryptic digestion, 1-40 from carboxypeptidase $Y$ digestion, and synthetic fragment 41-49 were not immunoreactive. The antiserum could detect the 15 residue (35-49) (Fig. 2, curve B) and the 11 residue (39-49) (Fig. 2, curve C) synthetic

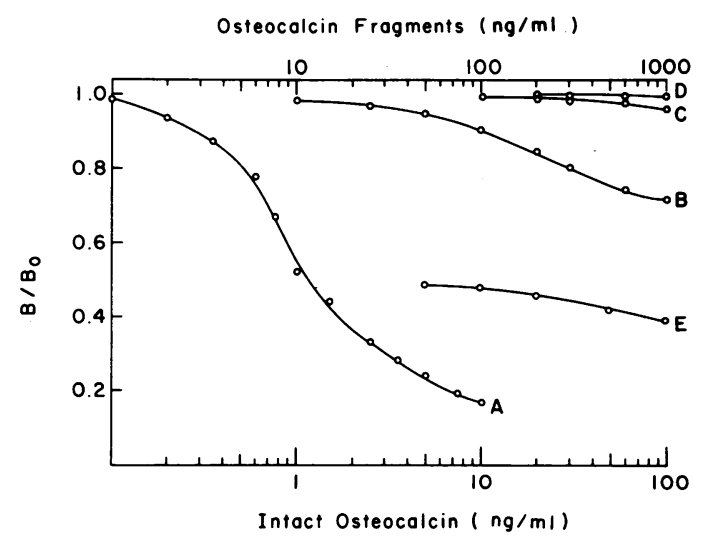

Figure 2. Relative fraction of radiolabeled osteocalcin bound to antibody in the presence of increasing concentrations of intact osteocalcin or fragments. $\mathrm{B} / \mathrm{Bo}=$ fraction of initial binding; $\mathrm{A}=$ purified intact bovine osteocalcin from 0 to $10 \mathrm{ng} / \mathrm{ml}$; $B=$ fragment $35-49,10$ to $1000 \mathrm{ng} / \mathrm{ml} ; \mathrm{C}$ and $\mathrm{D}$ are fragments $39-49$ and $41-49$, respectively, 100 to $1000 \mathrm{ng} / \mathrm{ml} ; \mathrm{E}=50$ to $1000 \mathrm{ng} / \mathrm{ml}$ of the $35-49$ fragment added to $1 \mathrm{ng} / \mathrm{ml}$ of purified intact osteocalcin showing that the apparent reactivities are additive. fragments but only at high concentrations (1,500 and 10,000 times the intact molecule, respectively). Additive immunoreactivity was found when increasing concentrations of fragment $35-49$ were combined with $1 \mathrm{ng} / \mathrm{ml}$ of purified bovine osteocalcin (Fig. 2, curve E).

Chromatography. Immunoreactive osteocalcin from healthy adults or dialysis patients eluted from Sephadex G-100 in a single peak. Subsequent HPLC of the G-100 peak obtained from the normal adults eluted as a single sharp peak in three separate experiments (Fig. 3). Bio-Gel P-10 chromatography of either of the serum pools from the dialysis patients resulted in a sharp immunoreactive peak eluting in the position of intact osteocalcin and a second broad peak with apparent molecular mass of 3,500 to 5,500 D (Fig. 4, solid circles). Multiple immunoreactive forms of osteocalcin were detected when these samples were separated by HPLC (Fig. 5). In addition to a peak that coeluted with intact osteocalcin and one that eluted afterwards, two peaks were found that eluted before intact osteocalcin when serum from patients with low osteoclastic resorptive surfaces was chromatographed and four peaks were detected before the intact peptide in the serum from patients with high osteoclastic resorptive surfaces. All the synthetic and enzymatically prepared fragments eluted before intact osteocalcin.

\section{Discussion}

In nonazotemic women with osteoporosis, serum osteocalcin levels correlate with bone formation rates but not with bone resorption (11). Our studies demonstrate that in dialysis patients, osteocalcin measurements in serum are related to both the rate of bone formation (Fig. 1) and the extent of osteoclastic bone resorption. The conclusion that the relationship between osteocalcin levels and bone resorption is independent of the coupling of bone formation and resorption, is supported by the chromatographic elution profile (Fig. 5). Multiple immunoreactive forms of osteocalcin are present in uremic serum. The second broad peak of immunoreactive osteocalcin eluting from Bio-Gel P-10 (Fig. 4) indicates that these multiple forms are smaller than the intact molecule. Although these fragments are rapidly cleared from serum and cannot be detected when renal function is normal (Fig. 3), the fragments accumulate in renal osteodystrophy and contribute to the circulating immunoreactivity in uremia (Fig. 5).

Osteocalcin fragments $1-19,20-43,45-49$, and 1-40 were not immunoreactive, whereas fragments $39-49$ and 35-49 were detected. The lower limits of detection for the 15 and 11 residue

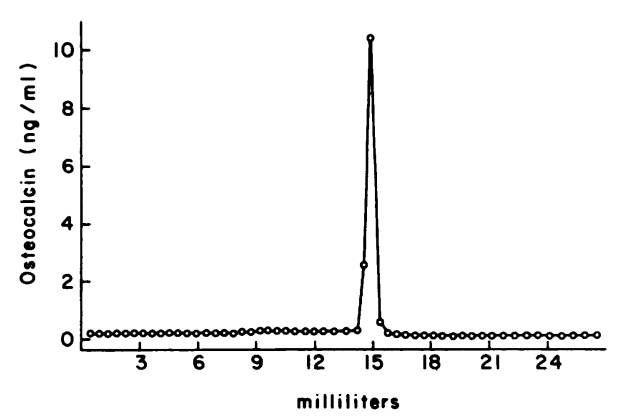

Figure 3. HPLC of immunoreactive osteocalcin from the serum of a normal adult after initial gel filtration. 


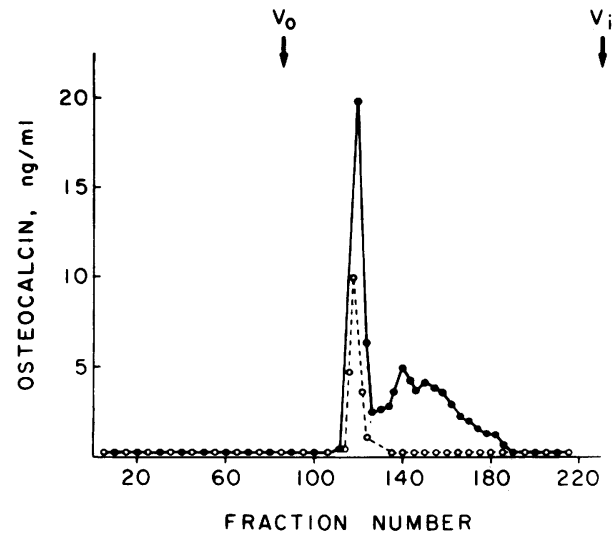

Figure 4. Bio-Gel P-10 filtration profile of normal serum is shown by the open circles and the profile of pooled sera from the dialysis patients by the closed circles. (The P-10 profile of the two dialysis groups was similar.) $\mathrm{Vo}=$ void volume; $\mathrm{Vi}=$ included volume.

carboxy-terminal peptides were 25 and $1,000 \mathrm{ng} / \mathrm{ml}$, respectively. This suggests that the fragments that we observed with the radioimmunoassay in uremic sera are derived from the carboxyterminal region of the protein and that the avidity of the antibody depends on fragment length. Other circulating fragments may be present in uremic serum but were not detected by our assay.

We cannot rule out other potential explanations for the appearance of the additional immunoreactive peaks in dialysis patients. First, fragments of osteocalcin could be produced by peripheral metabolism of the intact molecule. A second possibility

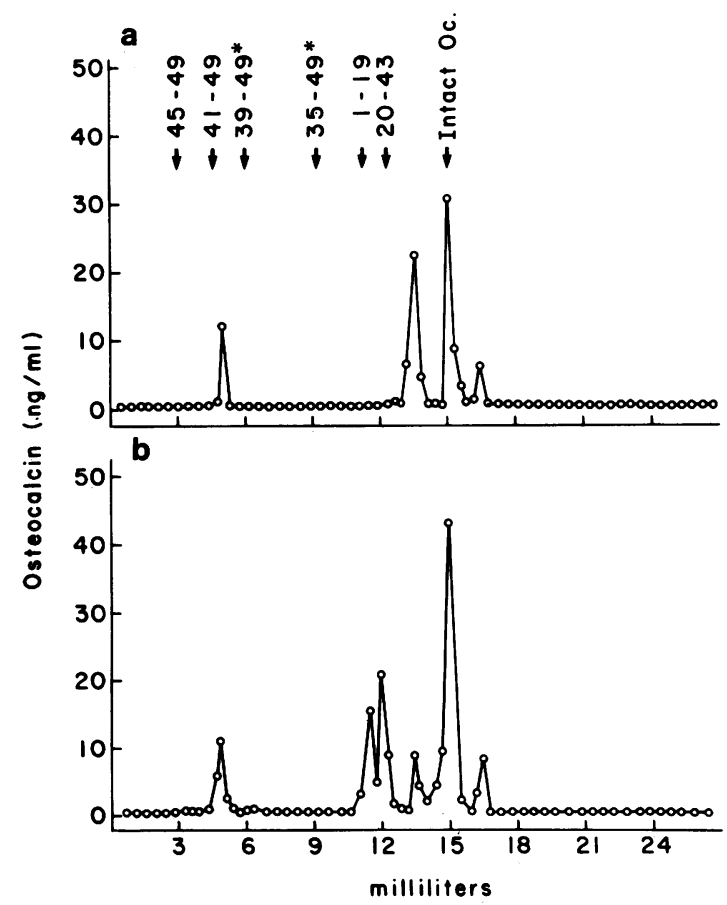

Figure 5. HPLC of immunoreactive osteocalcin from dialysis patients. Arrows indicate the elution positions of purified human osteocalcin and synthetic or enzymatic fragments of bovine osteocalcin. Asterisks indicate the immunogenic fragments. $\mathrm{Oc}=$ osteocalcin. (a) Patients $13,14,16,17$, and 18 with low osteoclastic resorptive surfaces. (b) $\mathrm{Pa}$ tients $1,3,7,8$, and 11 with high osteoclastic resorptive surfaces. is that during the accelerated bone formation of renal osteodystrophy, the synthesis of osteocalcin may be altered. Third, in uremia, circulating osteocalcin may become nonenzymatically carbamylated by the condensation of urea-derived cyanate with amino groups. Similar carbamylation occurs with hemoglobin and is proportional to the blood urea concentration (27). Gundberg and Gallop (28) have shown that in bone osteocalcin can become nonenzymatically glycosylated and it is possible that osteocalcin can also become carbamylated. Furthermore, the single peak that eluted following intact osteocalcin in our HPLC system (Fig. 5) has the same retention time as synthetic carbamylated osteocalcin (data not shown).

Our concept of the metabolism of osteocalcin is illustrated in Fig. 6. Osteocalcin is synthesized in the osteoblast (2) and is subject to stimulation by $1,25(\mathrm{OH})_{2} \mathrm{D}(29)$. Gla appears in osteocalcin as a result of posttranslational vitamin $\mathrm{K}$-dependent carboxylation of the glutamyl residues (2). Binding to calcium ions induces a conformational change in the molecule, which anchors osteocalcin to hydroxyapatite (5), so that most of the osteocalcin synthesized by osteoblasts remains in bone. Some osteocalcin escapes the bone and can be detected in the circulation by radioimmunoassay (6). When bone is resorbed, osteoclasts liberate osteocalcin fragments. With normal renal function, osteocalcin and these fragments are rapidly cleared by the kidney and contribute to the urine free Gla. In renal failure, these fragments accumulate and are detectable. The molecular heterogeneity of circulating osteocalcin is reminiscent of that of immunoreactive parathyroid hormone in uremic patients (30). The results of the present study suggest that differences in reports from various laboratories may reflect the sensitivity of the individual antibody for the circulating fragments of osteocalcin and that the radioimmunoassay is only valid as an index of bone formation in the absence of renal insufficiency. With appropriate monoclonal antibodies, it could be possible in the future to distinguish intact from specific fragments of osteocalcin and precisely differentiate between bone formation and resorption.

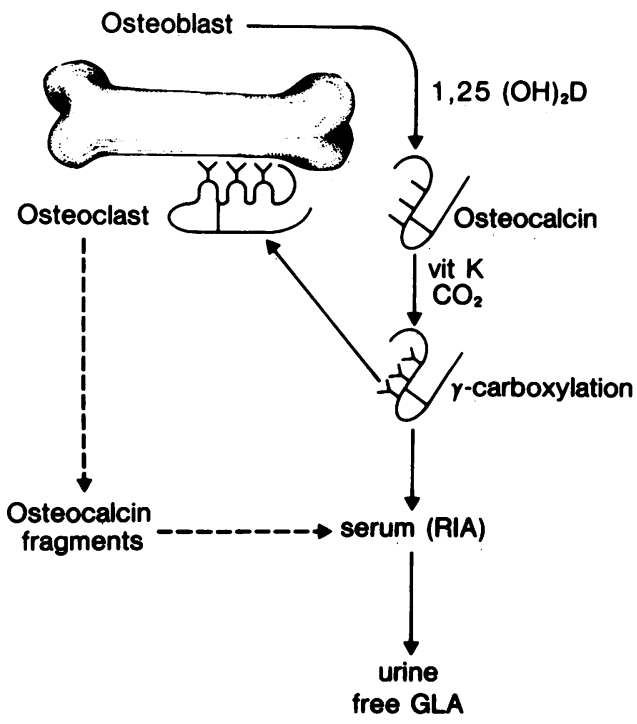

Figure 6. Proposed metabolism of osteocalcin. 1,25(OH) $)_{2} \mathrm{D}, 1,25$-dihydroxyvitamin $\mathrm{D}$; vit $\mathrm{K}$, vitamin $\mathrm{K} ; \mathrm{CO}_{2}$, carbon dioxide; RIA, radioimmunoassay. 


\section{Acknowledgments}

We thank Dr. Ken King for providing synthetic fragments of osteocalcin and Alex Liu, Jenifer Aronoff, Linda Sappington, and Marion Hutson for technical assistance. We are grateful to Drs. Paul Gallop, Peter Hauschka, Tom Carpenter, Ralph Kolbeck, and Ted Bransome for their helpful comments.

This work was supported in part by grants AG04727 and AM35407 from the National Institutes of Health.

\section{References}

1. Butler, W. T. 1984. Matrix macromolecules of bone and dentin. Collagen Rel. Res. 4:297-307.

2. Lian, J. B., and P. A. Friedman. 1978. The vitamin K-dependent synthesis of $\gamma$-carboxyglutamic acid by bone microsomes. J. Biol. Chem. 253:6623-6626.

3. Gallop, P. M., J. B. Lian, and P. V. Hauschka. 1980. Carboxylated calcium-binding proteins and vitamin K. N. Engl. J. Med. 302:14601466.

4. Hauschka, P. V., J. B. Lian, and P. M. Gallop. 1975. Direct identification of the calcium-binding amino acid $\gamma$-carboxyglutamate, in mineralized tissue. Proc. Natl. Acad. Sci. USA. 72:3925-3929.

5. Hauschka, P. V., and P. M. Gallop. 1977. Purification and calciumbinding properties of osteocalcin, the $\gamma$-carboxyglutamate-containing protein of bone. In Calcium Binding Proteins and Calcium Function. R. H. Wasserman, R. A. Corradino, E. Carafoli, R. H. Kaetsinger, D. H. MacLennan, F. L. Siegel, editors. Elsevier/North Holland, Amsterdam. 338-347.

6. Price, P. A., and S. K. Nishimoto. 1980. Radioimmunoassay for the vitamin K-dependent protein of bone and its discovery in plasma. Proc. Natl. Acad. Sci. USA. 77:2234-2238.

7. Price, P. A., J. G. Parthemore, and L. J. Deftos. 1980. New biochemical marker for bone metabolism. J. Clin. Invest. 66:878-883.

8. Deftos, L. J., J. G. Parthemore, and P. A. Price. 1982. Changes in plasma bone Gla protein during treatment of bone disease. Calcif. Tissue Int. 34:121-124.

9. Gundberg, C. M., J. B. Lian, P. M. Gallop, and J. J. Steinberg. 1982. $\gamma$-carboxyglutamic acid and serum osteocalcin as bone markers: studies in osteoporosis and Paget's disease. J. Clin. Endocrinol. Metab. 57:1221-1225.

10. Slovik, D. M., C. M. Gundberg, R. M. Neer, and J. B. Lian. 1984. Clinical evaluation of bone turnover by serum osteocalcin measurements in a hospital setting. J. Clin. Endocrinol. Metab. 59:228-230.

11. Brown, J. P., L. Malaval, M. C. Chapuy, P. D. Delmas, C. Edouard, and P. J. Meunier. 1984. Serum bone Gla protein: a specific marker for bone formation in postmenopausal osteoporosis. Lancet. i: 1091-1093.

12. Price, P. A., M. K. Williamson, and J. W. Lothringer. 1981. Origin of the vitamin $\mathrm{K}$-dependent protein found in plasma and its clearance by kidney and bone. J. Biol. Chem. 256:12760-12766.

13. Delmas, P. D., D. M. Wilson, K. G. Mann, and B. L. Riggs.
1983. Effect of renal function on plasma levels of bone Gla-protein. $J$. Clin. Endocrinol. Metab. 57:1028-1030.

14. Malluche, H. M., M. C. Faugere, P. Fanti, and P. A. Price. 1984. Plasma levels of bone Gla-protein reflect bone formation in patients on chronic maintenance dialysis. Kidney Int. 26:869-874.

15. Weinstein, R. S., G. F. Bryce, L. J. Sappington, D. W. King, and B. B. Gallagher. 1984. Decreased serum ionized calcium and normal vitamin D metabolite levels with anticonvulsant drug treatment. J. Clin. Endocrinol. Metab. 58:1003-1009.

16. Weinstein, R. S., J. L. Underwood, M. S. Hutson, and H. F. DeLuca. 1984. Bone histomorphometry in vitamin D-deficient rats infused with calcium and phosphorus. Am. J. Physiol. 246:E499-E505.

17. Schwartz, M. P., and R. R. Recker. 1981. Comparison of surface density and volume of human iliac trabecular bone measured directly and by applied stereology. Calcif. Tissue Int. 33:561-565.

18. Merz, W. A., and R. K. Schenk. 1970. A quantitative histological study on bone formation in human cancellous bone. Acta. Anat. 76:1115.

19. Maloney, N. A., S. M. Ott, A. C. Alfrey, N. L. Miller, J. W. Coburn, and D. J. Sherrard. 1982. Histological quantitation of aluminum in iliac bone from patients with renal failure. J. Lab. Clin. Med. 99:206216.

20. Frost, H. M. 1983. Bone histomorphometry: analysis of trabecular bone dynamics. In Bone Histormorphometry: Techniques and Interpretation. R. R. Recker, editor. CRC Press, Boca Raton, FL. 109-131.

21. Simon, L. S., A. M. Parfitt, A. R. Villaneuva, and S. M. Krane. 1984. Procollagen type I carboxyterminal extension peptide (pColl-I-C) in serum as a marker of collagen synthesis: correlation with iliac trabecular bone formation rate. Calcif. Tissue Int. 36:498 (Abstr.).

22. Gundberg, C. M., P. V. Hauschka, J. B. Lian, and P. M. Gallop. 1984. Osteocalcin: isolation, characterization, and detection. Methods Enzymol. 107:517-543.

23. Merrifield, R. B. 1964. Solid state peptide synthesis. I: The synthesis of a tetrapeptide. J. Am. Chem. Soc. 85:2149-2154.

24. Hauschka, P. V., J. Frenkel, R. DeMuth, and C. M. Gundberg. 1983. Presence of osteocalcin and related higher molecular weight $\gamma$ carboxyglutamic acid-containing proteins in developing bone. J. Biol. Chem. 258:176-182.

25. Edwards, A. L. 1979. Multiple Regression and the Analysis of Variance and Covariance. W. H. Freeman and Co., San Francisco, CA. $7-53$.

26. Teitelbaum, S. L. 1984. Progress in pathology: renal osteodystrophy. Human Pathol. 15:306-323.

27. Fluckiger, R., W. Harmon, W. Meier, S. Loo, and K. H. Gabbay. 1981. Hemoglobin carbamylation in uremia. N. Engl. J. Med. 304:823827.

28. Gundberg, C. M., and P. M. Gallop. 1984. Nonenzymatic glycosylation of osteocalcin in vivo. Calcif. Tissue Int. 36:486 (Abstr.).

29. Price, P. A., and S. A. Baukol. 1980. 1,25-dihydroxyvitamin $D_{3}$ increases synthesis of the vitamin K-dependent bone protein by osteosarcoma cells. J. Biol. Chem. 255:11660-11663.

30. Canterbury, J. M., and E. Reiss. 1972. Multiple immunoreactive molecular forms of parathyroid hormone in human serum. Proc. Soc. Exp. Biol. Med. 140:1393-1398. 